\title{
Influence of postpartum shock controllability on subsequent maternal behavior in rats
}

\author{
JON L. WILLIAMS \\ Kenyon College, Gambier, Ohio
}

\begin{abstract}
Responses of mother rats were observed $24 \mathrm{~h}$ before and 24 and $72 \mathrm{~h}$ after exposure to one of three 8-day postpartum treatments: shock escape training, yoked inescapable shock, or restrained with no shock. In contrast to those in the other two groups, the dams given inescapable shock showed slower speed to approach the nest, shorter durations of being on the nest, and lower frequency and shorter total duration of oral contact with their pups. These dams also retrieved their pups less frequently, but this measure, as well as the frequency of leaving the nest, did not result in significant differences between groups. Since the traditional interpretations of the learned-helplessness effect were not entirely able to account for these findings, the observed uncontrollable-stress-produced changes in maternal behavior were examined from an ethological perspective.
\end{abstract}

The fact that exposure to uncontrollable, as opposed to controllable, stress can have debilitating behavioral and physiological consequences has generated much research and theoretical interest (Maier \& Seligman, 1976; Sklar \& Anisman, 1981). Experiencing inescapable shock has been shown to interfere with the subsequent learning of escape responses (e.g., Anisman, Suissa, \& Sklar, 1980; Maier, Albin, \& Testa, 1973; Overmier \& Seligman, 1967) and appetitive operants (Rosellini, 1978; Rosellini, DeCola, \& Shapiro, 1982). In addition, transsituational deficits produced by inescapable shock have been found in tests of nonassociative or unlearned responses, such as nonreinforced shuttlebox running (e.g., Maier, Coon, McDaniel, Jackson, \& Grau, 1979), shockelicited fighting (e.g., Anderson, Crowell, Wikoff, \& Lupo, 1980), general activity (Drugan \& Maier, 1982), certain forms of exploratory behaviors (e.g., Bruto \& Anisman, 1983), and reactions to nociceptive stimuli (e.g., Jackson, Maier, \& Coon, 1979). These debilitations in behavior have been referred to as the learned helplessness effect, because they do not follow exposure to equivalent amounts of escapable shock and thus are a product of the uncontrollability of the shock as a stressor.

Several hypotheses have been offered to account for the learned-helplessness effect. According to Maier and Seligman (1976), inescapable shock results in the subject's learning that shock termination and its own behavior are independent events. As a result,

This research was supported by a USPHS Research Grant, 1 R03MH38528-01, to the author. The author wishes to thank Steven Maier, Thomas Minor, Charles Rice, and Kenneth King for their helpful comments. Reprint requests should be addressed to Jon L. Williams, Department of Psychology, Kenyon College, Gambier, $\mathrm{OH} 43022$. such organisms have difficulty learning responsereinforcer contingencies and also suffer from decreased motivation. As an alternative to this cognitive position, several investigators have argued that a motor response of remaining inactive is learned during exposure to inescapable shock and that this reduced movement competes with the acquisition and performance of subsequent responses that are motivated by aversive stimuli (e.g., Anderson, Crowell, Cunningham, \& Lupo, 1979; Bracewell \& Black, 1974; Glazer \& Weiss, 1976). A variant of the learned competing-inactivity hypothesis is that uncontrollable stress results in brain neurochemical depletions (e.g., norepinephrine and dopamine) which prevent the animal from performing later escape behaviors that involve sustained activity (Anisman, Remington, \& Sklar, 1979; Anisman \& Sklar, 1979). Anisman and Zacharko (1982) have recently argued that untrollable stressors hinder escape performance by disrupting response maintenance and that shock interruption or a novel cue can minimize escape deficits by altering the course of decline of shock-elicited activity. Although none of the above interpretations has been able to give a precise account of all the effects of inescapable shock, they are not incompatible with each other and the applicability of a given position seems to depend upon the specific tasks, procedures, and stress parameters that are being employed.

Virtually all of the published research examining the variables and the theoretical mechanisms related to the learned-helplessness effect, has involved testing somewhat arbitrary responses in contrived laboratory environments. To a large degree, interpretations of the learned-helplessness phenomenon reflect this focus. The major objective of the present investigator's current research is to utilize the traditional helplessness procedure in order to increase 
our understanding of how animals react to various stress conditions in a laboratory environment that facilitates the occurrence of more natural, speciestypical behaviors. For example, it has been noted that inescapable shock has striking effects on the agonistic behaviors of rats housed in mixed-sex colonies (Williams, 1982). Significant decreases in aggression and increases in defensive behaviors occurred when previously dominant rats were given inescapable (yoked) shocks and later retested by introducing conspecific intruders into their colonies. In contrast, no changes in agonistic behaviors and dominance were found after alpha rats received escapable shock or no-shock treatments.

The present experiment examines the influence of controllable versus uncontrollable shock on the maternal responses of mother rats as another class of species-typical behavior. For the past two decades numerous studies have examined the behavioral and physiological effects on the offspring when mother rats or mice were stressed during infancy (e.g., Denenberg \& Rosenberg, 1967), prior to mating (e.g., Anderson \& Ressler, 1973), during gestation (e.g., Chapman \& Stern, 1979), or postpartum (e.g., Ressler \& Anderson, 1973). While the types of stressors, experimental procedures, and control conditions used in these studies have been diverse, the general conclusion from this research is that prenatal as well as postnatal maternal stress produces increased emotionality in the offspring.

Although considerable attention has been paid to the effects of maternal stress on the development of offspring, very few studies have systematically investigated the influence that fear or stress has on maternal behavior, per se. Deni and Vocino (1980) reported that presentations of a conditioned signal, which has been paired with shock in an operant chamber 1 week prepartum, suppressed specific maternal behaviors (i.e., pup licking and time on nest) observed in the home cage when the pups were 1 week old. These transsituational effects reported by Deni and Vocino, in conjunction with the learnedhelplessness findings, raise a number of interesting questions. What would have been the effects of stress on maternal behavior if shock presentations were given 1 week postpartum and the behavioral observations were made on the following day? Even more pertinent to the present study, would unsignaled shock produce differential effects on certain maternal responses depending on whether it was escapable or inescapable? The present experiment represents the first reported work that examines the role of controllable versus uncontrollable postpartum stress on subsequent maternal caretaking of pups. Specifically, maternal responses in mother rats were observed $24 \mathrm{~h}$ before and 24 and $72 \mathrm{~h}$ after one of the following 8-day postpartum treatments: shock escape training, yoked inescapable shock, or restraint without shock.
The behavioral observations in this experiment were made after the dams were briefly removed from their pups and the home cage was placed in an observation chamber to prevent the influence of various extraneous stimuli. It was also assumed that the mild stress, produced by being separated from the pups and then observed in a relatively novel environment, might potentiate possible differential effects produced by the three experimental treatments.

\section{METHOD}

\section{Subjects}

Thirty adult nulliparous female albino rats, which were offspring of Holtzman animals that had been bred in the investigator's laboratory, served as the source of potential subjects. They were given free access to food and water and were housed individually in home cages.

\section{Apparatus}

The 30 female rats (and their pups) were each housed in $20 \times$ $50 \times 40 \mathrm{~cm}$ propylene tub cages with stainless steel wire tops. Food and water were freely available from the wire tops. The floors of the cages were covered with ground corn-cob mesh as bedding material, which was changed every 3 weeks until parturition.

Observations and event recordings of a dam's maternal behavior were made by placing the home cage inside a large $(100 \times 40$ $\times 70 \mathrm{~cm}$ ) observation box that was ventilated by an exhaust fan and illuminated from the ceiling by a G.E. 313 incandescent bulb, $115 \mathrm{~V}$. A trained observer could see the wire top of the colony cage through a $9 \times 9 \mathrm{~cm}$ window located on the side wall near the ceiling of the observation box. The interior of the box was painted black, and possible visual distraction was minimized by taping a red filter over the small window.

A multichannel event recorder was used to chart the frequency, duration, and sequence of specific maternal behaviors that occurred during the observation sessions. The recording area was an air-conditioned quiet room. A desk lamp with a 20 -W bulb directed on the event. recorder was the only source of room illumination during an observation session.

Shock and restraint conditions were given in three wheel-turn boxes (measuring $15.5 \times 12.0 \times 17.0 \mathrm{~cm}$ ) located in an experimental cubicle. The side, front, and rear walls, as well as the top and floor of each chamber, were made of Plexiglas. A 64-cm-diam grooved Plexiglas wheel was located on the front wall and extended $1.5 \mathrm{~cm}$ into the chamber. The force required to move the wheel a quarter turn was approximately $30 \mathrm{~g}$. The rat's tail extended through a hole in the rear of each apparatus and was taped to a Plexiglas rod. In two of the three chambers, unscrambled shocks were delivered to the rat's tail via fixed electrodes from separate constant current Lafayette 82400 shock sources, which were continuously calibrated. Each chamber was housed in a soundattenuating box equipped with a white-noise speaker, a houselight, and a ventilating fan. All treatment contingencies were electronically controlled, and the wheel-turn latencies in the chamber used for escape training were automatically recorded via an electric printout timer.

Females were carried to and from the wheel-turn boxes in small $(20 \times 30 \times 40 \mathrm{~cm})$ plastic carrying tubs with bedding material on the floors. A separate tub was used for moving the females assigned to cach of the three treatment conditions, and this same tub was also used as a waiting area for the dams when the home cage containing her pups was placed inside the observation box.

\section{Procedure}

An experienced breeding male was housed with each female for 12-14 days and then removed from her home cage. Soon after parturition, each female was matched with two other females 
which had given birth to a litter of at least three pups within a 36-h period of each other. Each member of a triplet of dams was assigned randomly to one of three treatments: escape $(E)$, yoked $(Y)$, or restrained $(R)$ groups. In order to have female triplets with litters of a comparable age and number, it was not possible to use six of the original $\mathbf{3 0}$ rats in the actual experiment. The remaining 24 dams were assigned randomly to treatment groups of eight subjects each, and the mean number of pups per litter was $8.00,7.75$, and 8.63 for Groups $E, Y$, and $R$, respectively.

The initial ( $24 \mathrm{~h}$ pretreatment) 15 -min observation session of the females with their pups was conducted on Day 7 postpartum between 7:00 p.m. and midnight. Immediately before the session, the dam was removed from the colony cage and placed in the waiting tub, which was also to be used as the carrying cage for her particular group. The home cage containing the pups was then placed inside the observation box, with the food and water bottle removed from the wire top. Three minutes later, the experimenter checked to make sure that all of the pups were in the nest area (i.e., usually in one corner of the cage). Then the mother was removed from the waiting tub and placed in the corner of the home cage that was farthest from the nest. After the wire top of the home cage and the door to the observation box were closed, a trained observer used the multichannel event marker to record specific maternal responses.

An observation session for each female with her pups lasted $15 \mathrm{~min}$. Six measures were recorded: (1) latency to approach nesttime until the dam entered the nest area quadrant of the cage and remained there for a period of at least $10 \mathrm{sec}$, (2) number of times leaving nest-frequency of leaving nest area quadrant, (3) number of retrieving instances-frequency of the dam picking up and carrying a pup to the nest area, (4) duration in nest area-time spent in the nest-area quadrant, (5) number of oral contacts-frequency of oral-contact bouts of licking, nuzzling, or sniffing pups (cf. Mayer, Freedman, \& Rosenblatt, 1979) or simply touching a pup with the oral region (Deni \& Vocino, 1980), and (6) duration of oral contact-time spent engaging in oral-contact bouts with pups. Oral-contact bouts were not scored when the pups were picked up or carried. After the observation session, the home cage, containing the mother and her pups, was removed from the observation box and returned to the colony area.

One day after the initial observation test, triplets of dams were run according to their assigned treatment conditions: wheel-turn escape training (Group E), inescapable yoked shock (Group Y), and restraint without shock (Group $R$ ). To begin each training session, the female rat was placed in the assigned waiting tub and transported to the treatment room. Then the Group E females received one shock-escape training session in the wheel-turn box. The session consisted of 80 trials presented on a variable-time schedule, with a mean interval of $60 \mathrm{sec}$ and a range of 30-120 sec. Shock terminated when the rat had completed a one-quarter turn of the wheel beyond $.8 \mathrm{sec}$ following shock onset. Wheel turns during the first $.8 \mathrm{sec}$ of shock were considered to be unlearned shock-elicited responses, which would not function as an effective coping behavior against a helplessness effect (Maier \& Jackson, 1977). Therefore, such responses were programmed to have no consequence. In order to insure that the task exposed the animal to a critical duration of exposure to shock, the response delay interval after the onset of shock was increased from .8 to $1.0 \mathrm{sec}$ on Trial 20, to $1.3 \mathrm{sec}$ on Trial 40 , and to $1.6 \mathrm{sec}$ on Trial 60 . Shock presentations, delivered via tail electrodes, terminated after $30 \mathrm{sec}$ if an escape response had not occurred. The shock intensity for all rats in Groups $E$ and $Y$ was begun at $.8 \mathrm{~mA}$ and then increased to $1.0 \mathrm{~mA}$ on Trial 20 , to $1.3 \mathrm{~mA}$ on Trial 40 , and to 1.6 mA on Trial 60. This was done because previous studies (e.g., Maier \& Jackson, 1977) and other experiments by the author had revealed a deterioration of sustained wheel-turn performance over trials when the response-delay interval of shock termination was increased and the shock intensity remained at $1.0 \mathrm{~mA}$.

The subjects in Group $Y$ received the same number, duration, and intensity of shocks as did their Group E partners. Shock began simultaneously for both subjects and terminated whenever the partner rat in Group $E$ made the appropriate wheel-turn response. The subjects in Group $R$ were restrained in the wheel-turn box for an identical (i.e., $90 \mathrm{~min}$ ) period of time but were not shocked. Immediately after the triadic treatment, each of the three females was placed in the appropriate group's waiting tub cage for $5 \mathrm{~min}$, while the wheel-turn boxes were cleaned. After this period, the dams were transported in the waiting cage to the colony room and put into the home cage with their pups.

To determine the effects of the triadic treatment on the maternal behavior of the female rats, a $24-h$ posttreatment observation test was conducted using the same procedures as those in the 24-h pretreatment observation session. A test for possible long-term effects was given $48 \mathrm{~h}$ later, or $72 \mathrm{~h}$ after the triadic treatment session.

\section{RESULTS}

All eight dams in Group $E$ learned the wheel-turn escape response during the treatment phase. Their mean latencies decreased over the eight successive blocks of 10 acquisition trials. The mean response latency (minus the .8-sec response delay interval) on the first block of trials was $12.30 \mathrm{sec}$, with a range of 1.9-29.2 sec. This differed significantly from the mean latency (minus the 1.6-sec response delay interval) of $2.68 \mathrm{sec}$, with a range of $.80-5.80 \mathrm{sec}$, on the last block of trials [t(7) $=2.57, \mathrm{p}<.025]$.

All three groups had similar scores during the pretreatment observation session on the majority of maternal dependent measures. However, during the posttreatment sessions, Group Y showed a striking decrement in most of these behaviors and Groups $\mathbf{E}$ and $\mathbf{R}$ showed either similar or increased response levels. The changes in each response measure across successive pairs of observation sessions and the differences between groups were examined by means of two mixed-design ANOVAs (i.e., 24-h pretreatment vs. 24-h posttreatment sessions and 24-h vs. 72-h posttreatment sessions). All the main effects and interactions found to be statistically significant at the .05 level were further analyzed by a series of NewmanKeuls tests to determine whether the differences either within or between groups were significant. For each maternal measure, only the findings for which the Newman-Keuls test were significant (at either the .05 or the .01 level) are described.

Figure 1 presents the mean speed (i.e., reciprocal of the latency in seconds $\times 100$ ) with which the dams in the three treatment groups approached the nest, and remained there for a period of at least $10 \mathrm{sec}$, during each of the observation sessions. Speed scores were used in the statistical analyses because the raw latency scores were not normally distributed within groups. As can be seen from this figure, there was a significant interaction effect (groups $\times$ sessions) between speed to approach the nest area from the pretreatment to the 24-h posttreatment session $[F(2,21)$ $=6.59, \mathrm{p}<.011$. The mother rats in Group $Y$ showed a sharp $(p<.01)$ decline in speed of approach $24 \mathrm{~h}$ 


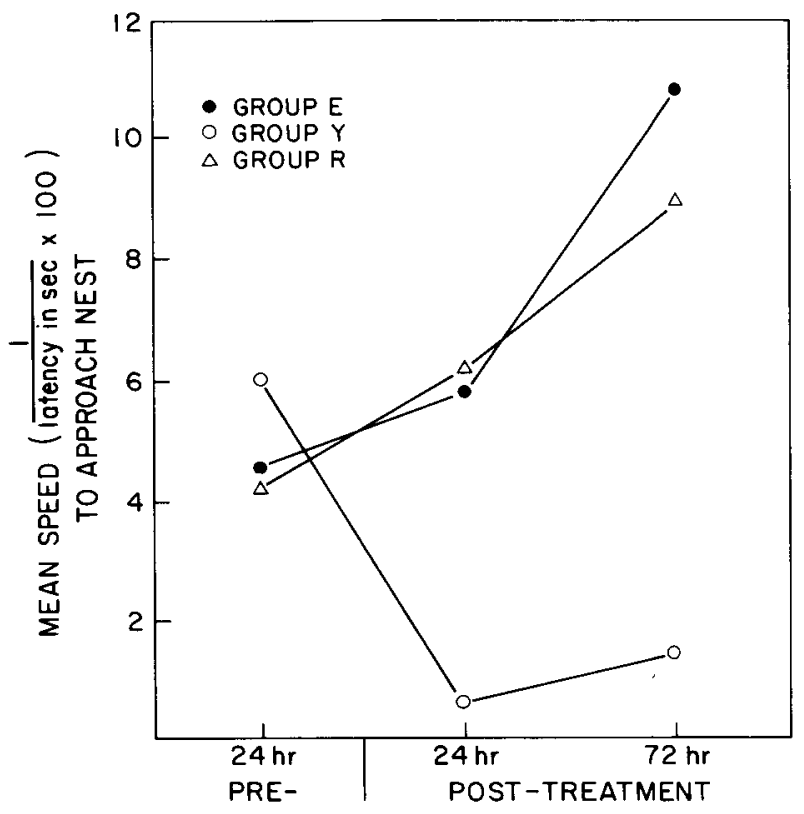

Figure 1. Mean speed (reciprocal of the latency in seconds $x$ 100) at which the dams approached the nest containing their pups at the beginning of the three observation sessions. The three treatment conditions are escapable shock (Group E), inescapable yoked shock (Group Y), and restrained without shock (Group R).

following the inescapable-shock treatment session. In contrast, dams that had experienced either escapable shock (Group E) or restraint (Group R) approached their nests and pups slightly faster during the 24-h posttreatment session. The long-term assessment of the treatment conditions, which is also shown in Figure 1, clearly reveals that there is a difference between the groups $[F(2,21)=6.63, p<.01]$ and between the 24-h versus the 72-h posttreatment sessions $[F(1,21)=4.34, p<.05]$. Relative to the subjects in the other two groups, the rats in Group $Y$ continued to approach the nest area very slowly on both sessions $(p<.01)$. On the other hand, the rats in Groups $E$ and $R$ approached the nest faster during the final session, and the former group's increment was found to be statistically significant $(p<.05)$.

Figure 2 presents the mean total duration per session that the dams remained in the nest-quadrant area of the home cage. It is important to realize that the total-duration data shown in this figure are confounded by the significant differences in the latency to approach the nest (see Figure 1). Nevertheless, the duration-in-nest-area measure is important to examine because it obviously determines the upper limit for the number and duration of oral contacts that a dam could show during a given session. The statistical analyses of the nest-duration data revealed a significant interaction between groups $\times$ pre-posttreatment sessions $[F(2,21)=9.30, p<.01]$. Group $E$ showed a significant $(p<.01)$ increase in nest duration one day following exposure to escapable shock, whereas Group $Y$ subjects decreased the time they spent on the nest after an experience with yoked inescapable shock $(p<.01)$. The main effect between groups during the two posttreatment sessions was also found to be significant $[\mathrm{F}(2,21)=5.84, \mathrm{p}<.01]$. During the final session, the three groups partially returned to their respective pretreatment levels, although the groups $\times$ posttreatment sessions interaction was not significant.

Because of the previously described group differences in the latency to approach the nest and the nest-duration measure, pup-licking or oral contact by the dams was examined in terms of the number of contacts per minute while each dam was actually in the nest area. The rate of maternal oral contacts with pups is shown in Figure 3 for each group across the observation sessions. As can be seen, the rate of oral contacts showed the same general configuration of results that was observed for the two previously described measures; there was a significant main effect of pretreatment versus 24 -h posttreatment $[F(2,21)$ $=4.90, \mathrm{p}<.05]$ and a significant groups $\times$ preposttreatment interaction effect $[\mathrm{F}(2,21)=10.14$, $p<.01]$. These effects were primarily due to the fact that Group Y showed a significant $(p<.01)$ decrease in rate of oral contacts after the treatment, whereas both Group $E$ and Group $R$ increased their rate of

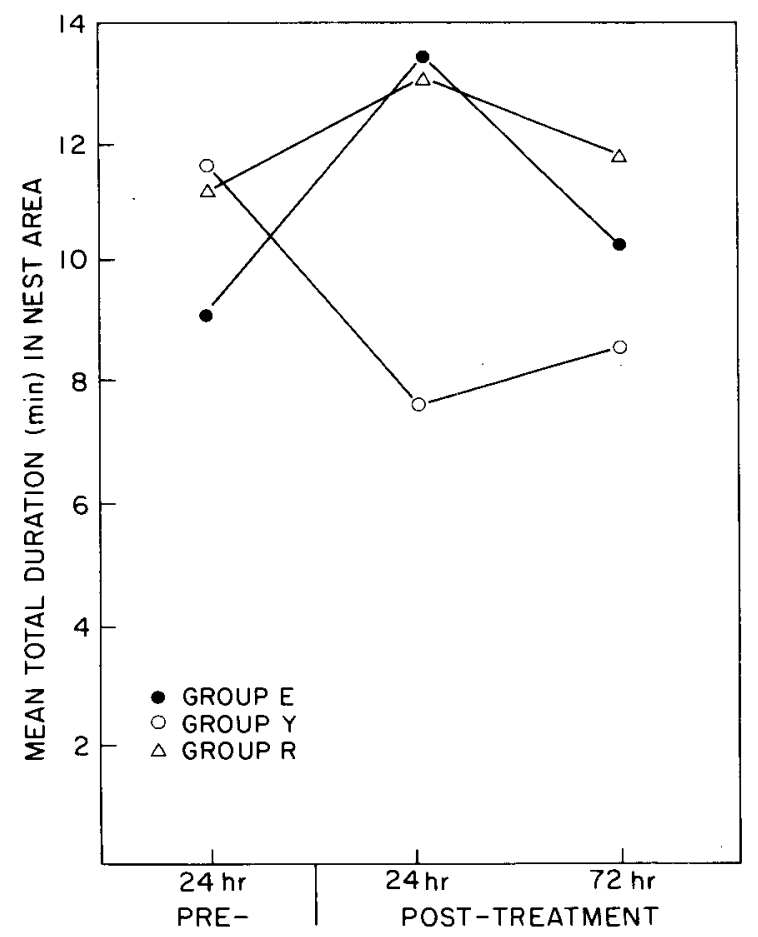

Figure 2. Mean total duration (minutes) that the dams were in the nest quadrant area of the home cage during the three observation seasions. The three treatiment conditions are escapable shock (Group E), inescapable yoked shock (Group Y), and restrained without shock (Group R). 


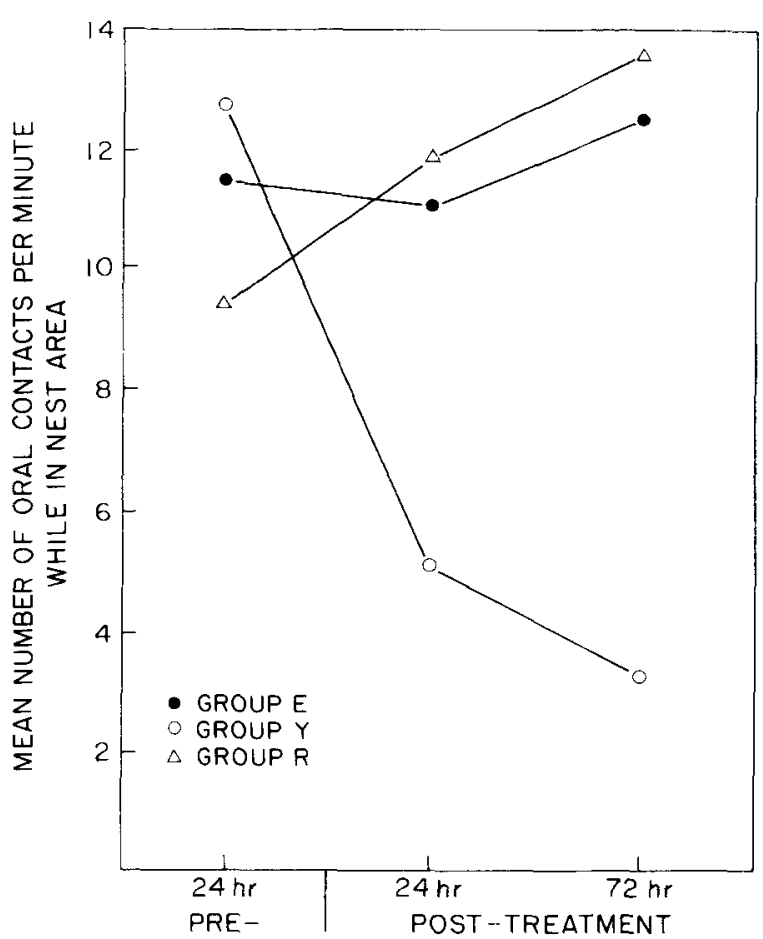

Figure 3. Mean number of oral contacts per minute that the dams had with their pups while in the nest area during the three observation sessions. The three treatment conditions are escapable shock (Group E), inescapable yoked shock (Group Y), and restrained without shock (Group R).

contacts. The difference between Group $Y$ and Groups $E$ and $R$ remained significant $(p<.01)$ during both of the posttreatment sessions.

A similar set of findings was revealed when the results of the total duration of oral contacts with the pups were analyzed. The groups $\times$ pre-posttreatment session interaction of total contact duration was significant $[F(2,21)=6.63, p<.01]$, with Groups $E$ and $R$ increasing $(p<.01)$ their total duration of contact after the treatment and Group $Y$ showing a decrease in duration $(p<.01)$. Finally, during the final 72-h posttreatment session, Group Y continued to show a lower total duration of oral contacts with pups than did Groups $E$ and $R(p<.01)$. Further analyses of these data indicated that each oral-contact bout was consistently from 3 to $5 \mathrm{sec}$, regardless of the subject's assigned group, and that there were significant positive correlations within each group (rs of .71, $.85, .82$ for Groups $E, Y$, and $R$, respectively) between the number of oral contacts and the total duration per session of engaging in this type of behavior.

No significant main effects or interactions were observed in the frequency of leaving the nest area or in the number of pup-retrieving instances. However, from the pretreatment to the 24-h posttreatment sessions, Group Y showed a definite decrease $(p<.10)$ in pup retrieving. This decrement was only tempo- rary, and the number of retrieving instances recovered to baseline in the 72-h posttreatment session.

\section{DISCUSSION}

Inescapable yoked shock, as opposed to escapable shock, produced clear decrements in terms of (1) the speed with which mothers approached the nest, (2) the time they spent in the vicinity of the nest, and (3) the frequency and total duration of oral contacts they had with their offspring while they were in the nest area. These disruptions in maternal responses from the pretreatment observation session were found during both the 24- $h$ and the 72-h posttreatment sessions for mothers in Group Y. In contrast, the dams in Groups $E$ and $R$ showed an increment in these maternal behaviors during the course of the three successive observation sessions. Finally, no significant changes, either between groups or over sessions, were observed for the frequency of leaving the nest area or the number of pup-retrieving instances. These latter two measures were simply not sensitive to the stress manipulations used in the present study. Deni and Vocino (1980) noted that the frequency of leaving the nest and of retrieving pups did not change significantly when mother rats were exposed to a signal that had been previously paired with shock. Consistent with the present findings, these investigators also observed that duration-on-nest and oral contact measures were definitely influenced by shock-fear manipulations.

It is not clear what mechanism or theory that has been proposed to account for the learned-helplessness effect during aversive testing can best explain the long-term disruption of many of the maternal responses that were noted in this study. For example, the proponents of the learned-inactivity or competingresponse hypothesis assume that the actual presence of shock in most helplessness studies mediates the transfer of competing forms of behaviors from the inescapable to the subsequent aversive test situation (e.g., Anderson et al., 1979). However, there was no shock or specific aversive stimulus present during the posttriadic observations of maternal behavior in the present experiment. This fact also poses some difficulty for a norepinephrine-depletion account of these data. According to Anisman and his colleagues (e.g., Anisman \& Sklar, 1979), inescapable shock produces an amine depletion, which in turn results in a disruption of response maintenance when animals are later exposed to shock during an escape-learning session. Again, this view clearly assumes that aversive stimulation must be present in the test environment in order for prior exposure to inescapable shock to have a debilitating effect. Although no aversive stimulation (i.e., shock) was specifically administered during the maternal observation sessions of this study, one could argue that briefly separating the 
mother from her pups before observing her maternal behavior in a somewhat novel environment was a successful experience. However, it remains to be shown that this type of stressor, during testing, is sufficiently intense or similar enough to the experience of receiving prior inescapable shock that it could elicit competing responses or reinstate neurochemical/ hormonal variations in norepinephrine, dopamine, serotonin, prolactin, ACTH, the endorphins, etc.

While it is somewhat premature to speculate as to which of the above neurochemical/hormonal alternations may have been responsible for the observed effects on maternal behavior, the possibility of reinstated prolactin changes definitely deserves further study. For some time, it has been known that increased prolactin concentrations maintain an elevated heat load in lactating dams, which make them vulnerable to further increases in temperature and eventually force them to terminate contact bouts (i.e., huddling) with their pups (Woodside, Leon, Attard, Feder, Siegel, \& Fischette, 1981). Recently, Telner, Merali, and Singhal (1983) have reported that plasma prolactin in male rats is influenced by the controllability of shock as a stressor. Furthermore, like the sensitivity of amine depletions to the controllability of shock, these differential prolactin reactions were found to have a rapid time course. Therefore, some type of reinstatement or sensitization process would have to be postulated in order to explain the present results in terms of changes in the level of prolactin.

Relative to the above positions, the present findings are perhaps more consistent with the learnedhelplessness theory (Maier \& Seligman, 1976). According to this view, inescapable aversive events produce cognitive, motivational, and emotional deficits which influence subsequent behaviors observed in completely different situations. While much of the previously reported research has focused on the cognitive and motivational disruptions produced by inescapable shock, there is some evidence to indicate that this experience also alters emotional and affective states (see Weiss, Goodman, Losito, Corrigan, Charry, \& Bailey, 1981). Assuming that such changes in affect are transitional, it is conceivable that inescapable shock might later have a significant influence on how a mother rat interacts with her offspring. This argument is, to some degree, consistent with the fact that differential affective states resulting from shock controllability have been observed in terms of the changes in aggressive and defensive behaviors seen when dominant colony males encounter conspecific intruders (Williams, 1982). However, the major weaknesses of the learned-helplessness interpretation are that (1) it does not specify a precise behavioral or physiological mechanism for why inescapable shock produces a deficit in affect, and (2) it fails to describe the boundary conditions or limits of the helplessness effects.
A useful strategy in the study of learned helplessness, particularly when investigating species-typical behaviors, is to adopt a more ethological perspective. For example, when pup-licking is examined in terms of resource exchange between mother and offspring, the observed suppression of this response following inescapable shock is consistent with a variation of the learned-helplessness theory. A 10-day postpartum female with a litter of 8 pups produces $42-48 \mathrm{ml}$ of milk (Friedman \& Bruno, 1976), which contains 31-35 ml of water. This water loss represents 30\%$35 \%$ of the female's total daily water intake (Friedman, Bruno, \& Alberts, 1981), and it places a severe strain on the female's body-fluid balance. However, by licking pup urine, mothers are able to reclaim $65 \%$ $70 \%$ of the fluids they had previously transferred to their young. Although lactating females continue to show considerable pup-licking when water is freely available from bottles in their home cages, females deprived of water increase anogenital licking of pups and presumably ingest more urine (Friedman et al., 1981). These findings suggest that pup-licking can function as a type of consummatory behavior, which may also be a heat-releasing mechanism. Assuming this is true, it is interesting to note that yoked rats under the triadic-shock procedure have been shown to later drink less water in their home cages than have escapably shocked or restrained control animals (Weiss, 1968). Thus, the differential effects that shock controllability has on consummatory behavior could be responsible for the striking differences that were found in pup-licking between the inescapably shocked versus the escape and restrained dams in this experiment. Furthermore, it could also be argued that these dams did not approach or stay in the nest area because they were less motivated to gain access to water by licking their offspring. This line of reasoning receives additional support from the recent finding that inescapable shock influences motivational/reward processes as measured by responding for electrical brain stimulation (Zacharko, Bowers, Kokkinidis, \& Anisman, 1983).

Another possible explanation of the present findings which stems from an ethological perspective is that the stress of inescapable shock may have influenced certain behaviors in the pups which, in turn, were important in initiating or maintaining maternalpup interactions. Although no systematic analysis was made of the pups' responses, it may have been that the pups did not nurse as frequently or as vigorously from the dams that had just received inescapable shock. Such altered behavior by the pups could have conceivably resulted in later changes in maternal behavior. Obviously, more research is needed to determine the specific factors, resulting from exposure to inescapable shock, that are able to disrupt the transactional relationship between mother and offspring. We are currently examining 
certain aspects of this problem by using pups that have been randomly selected from the litters of dams in all of the pretreatment conditions.

It is important to reemphasize that the observational procedure used in this study was somewhat atypical in that the dams were briefly separated from their pups and later their maternal behavior was recorded when the home cage was placed in a somewhat novel enclosure. While there is ample evidence that indicates that exposure to inescapable shock disrupts subsequent responses as well as neurochemical/hormonal processes, the time course of these effects is typically short unless reinstatement shocks are given just before testing. As mentioned before, it is conceivable that inescapable shock suppresses certain maternal behaviors for a prolonged period only under circumstances that evoke moderate to high levels of fear or arousal. Pilot research data from our laboratory, comparing the posttreatment maternal responses of dams in their familiar home cages versus novel environments, suggests that exposure to inescapable shock has a more disruptive effect in the latter situation. These preliminary results suggest that we must be cautious in making generalizations from the present data concerning normal maternal-pup interactions in a familiar environment. However, these findings should stimulate further research on the role that sensitization (and conditioning) processes might have in potentiating and prolonging the influence of uncontrollable stress on species-typical behaviors.

\section{REFERENCES}

Anderson, D. C., Crowell, C. R., Cunningham, C. L., \& LuPo, J. V. (1979). Behavior during shock exposure as a determinant of subsequent interference with shuttle box escapeavoidance learning in the rat. Journal of Experimental Psychology: Animal Behavior Processes, 5, 243-257.

Anderson, D. C., Croweli, C. R., Wikoff, M. B., \& Lupo, J. V. (1980). Activity during prior shock determines subsequent shock-elicited fighting in the rat. Animal Learning \& Behavior, 8, 664-672.

Anderson, L. T., \& Ressler, R. H. (1973). Response to a conditioned aversive event in mice as a function of frequency of premating maternal shock. Developmental Psychobiology, 6, 113-121.

Anisman, H., Reminaton, G., \& Sklar, L. S. (1979). Effects of inescapable shock on subsequent escape performance: Catecholaminergic and cholinergic mediation of response initiation and maintenance. Psychopharmacology, 61, 107-124.

Anisman, H., \& Skrar, L. (1979). Catecholamine depletion in mice upon reexposure to stress: Mediation of the escape deficits produced by inescapable shock. Journal of Comparative and Physiological Psychology, 93, 610-625.

Anisman, H., Sutssa, A., \& Sklar, L. S. (1980). Escape deficits induced by uncontrollable stress: Antagonism by dopamine and norepinephrine agonists. Behavioral and Neural Biology, 28, 34-47.

Anisman, H., \& Zacharko, R. M. (1982). Stimulus change influences escape performance: Deficits induced by uncontrollable stress and by haloparidol. Pharmacology, Biochemistry and Behavior, 17, 263-269.
Bracewell, R. J., \& Black, A. H. (1974). The effects of restraint and noncontingent pre-shock on subsequent escape learning in the rat. Learning and Motivation, 5, 53-69.

Bruto, V., \& ANisman, H. (1983). Alteration of exploratory patterns induced by uncontrollable shock. Behavioral and Neural Biology, 37, 302-316.

Chapman, R. H., \& Stern, J. M. (1979). Failure of severe maternal stress or ACTH during pregnancy to affect emotionality of male rat offspring: Implication of litter effects for prenatal studies. Developmental Psychobiology, 12, 255-267.

Deneberg, V. H., \& Rosenberg, K. M. (1967). Nongenetic transmission of information. Nature, 216, 549-550.

DEN1, R., \& Vocino, J. (1980). Changes in maternal behavior in rats during exposure to a stimulus predicting shock. Psychological Record, 30, 525-529.

Drugan, R. C., \& Maier, S. F. (1982). The nature of the activity deficit produced by inescapable shock. Animal Learning \& behavior, 10, 401-406.

Friedman, M. I., \& Bruno, J. P. (1976). Exchange of water during lactation. Science, 197, 409-410.

Friedman, M. I., Bruno, J. P., \& Alberts, J. R. (1981). Physiology and behavioral consequences in rats of water recycling during lactation. Journal of Comparative and Physiological Psychology, 95, 26-35.

Glazer, H. I., \& WEiss, J. M. (1976). Long-term interference effect: An alternative to "learned helplessness." Journal of Experimental Psychology: Animal Behavior Processes, 2, 201-213.

JaCkson, R. L., MAIER, S. F., \& Coon, D. J. (1979). Long-term analgesic effects of inescapable shock and learned helplessness. Science, 206, 91-94.

Mater, S. F., Albin, R. W., \& Testa, T. J. (1973). Failure to learn to escape in rats previously exposed to inescapable shock depends on nature of escape response. Journal of Comparative and Physiological Psychology, 85, 581-592.

Maier, S. F., Coon, D. J., McDaniel, M. A., Jackson, R. L., \& GraU, J. (1979). The time course of learned helplessness, inactivity, and nociceptive deficits in rats. Learning and Motivation, 10, 467-487.

MaIER, S. F., \& Jackson, R. L. (1977). The nature of the initial coping response and the learned helplessness effect. Animal Learning \& Behavior, 5, 407-414.

Maier, S. F., \& Seligman, M. E. P. (1976). Learned helplessness: Theory and evidence. Journal of Experimental Psychology: General, 105, 3-46.

Mayer, A. D., Freedman, N. C. G., \& Rosenblatt, J. S. (1979). Ontogeny of maternal behavior in the laboratory rat: Factors underlying changes in responsiveness from 30-90 days. Developmental Psychobiology, 12, 425-490.

Ovenmier, J. B., \& Seligman, M. E. P. (1967). Effects of inescapable shock upon subsequent escape and avoidance learning. Journal of Comparative and Physiological Psychology, 63, 23-33.

Ressler, R. H., \& Anderson, L. J, (1973). Avoidance conditioning in mice as a function of their mothers' training. Developmental Psychobiology, 6, 97-103.

Rosellini, R. A. (1978). Inescapable shock interferes with the acquisition of an appetitive operant. Animal Learning \& Behavior, 6, 155-159.

Rosellini, R. A., DeCola, J. P., \& Shapiro, N. R. (1982). Cross-motivational effects of inescapable shock are associative in nature. Journal of Experimental Psychology: Animal Behavior Processes, 8, 376-388.

Sklar, L. S., \& Anisman, H. (1981). Stress and cancer, Psychological Bulletin, 89, 369-406.

Telner, J. I., Merali, Z., \& Singhal, R. L. (1983). Timedependent changes in plasma prolactin level and stress controllability. Progress in Neuro-Psychopharmacology and Biological Psychiatry, 6, 459-462.

Weiss, J. M. (1968). Effects of coping responses on stress. Journal of Comparative and Physiological Psychology, 65, 251-260. 
Weiss, J. M., Goodman, P. A., Losito, B. G., Corrigan, S., Charry, J. M., \& Bailey, W. H. (1981). Behavioral depression produced by an uncontrollable stressor: Relationship to norepinephrine, dopamine and serotonin levels in various regions of rat brain. Brain Research Reviews, 3, 167-205.

WILLIAMS, J. L. (1982). Influence of shock controllability by dominant rats on subsequent attack and defensive behaviors toward colony intruders. Animal Learning \& Behavior, 10, 305-313.

Woodside, B., Leon, M., Attard, M., Feder, H. H., Siegel, H. I., \& Fischetre, C. (1981). Prolactin-steroid influences on the thermal basis for mother-young contact in Norway rats. Journal of Comparative and Physiological Psychology, 95, 771-780.

Zacharko, R. M., Bowers, W. J., Kokkinidis, L., \& Anisman, H. (1983). Region-specific reductions of intracranial selfstimulation after uncontrollable stress: Possible effects on reward processes. Behavioral Brain Research, 9, 129-142.

(Manuscript received June 10, 1983;

revision accepted for publication November 10, 1983.) 